\title{
SEOM clinical guidelines in early stage breast cancer (2018)
}

\author{
F. Ayala de la Peña ${ }^{1}$ - R. Andrés ${ }^{2} \cdot$ J. A. Garcia-Sáenz ${ }^{3} \cdot$ L. Manso ${ }^{4} \cdot$ M. Margelí $^{5} \cdot$ E. Dalmau $^{6} \cdot$ S. Pernas ${ }^{7} \cdot$ A. Prat $^{8}$. \\ S. Servitja ${ }^{9} \cdot$ E. Ciruelos ${ }^{10,11}$
}

Received: 17 October 2018 / Accepted: 19 October 2018 / Published online: 15 November 2018

(c) The Author(s) 2018

\begin{abstract}
Breast cancer is the most common cancer in women in our country and it is usually diagnosed in the early and potentially curable stages. Nevertheless, around $20-30 \%$ of patients will relapse despite appropriate locoregional and systemic therapies. A better knowledge of this disease is improving our ability to select the most appropriate therapy for each patient with a recent diagnosis of an early stage breast cancer, minimizing unnecessary toxicities and improving long-term efficacy.
\end{abstract}

Keywords Early breast cancer $\cdot$ Adjuvant $\cdot$ Neoadjuvant $\cdot$ Genomic predictive test $\cdot$ Follow-up

F. Ayala de la Peña

frayala@um.es

R. Andrés

andresraquelc@gmail.com

J. A. Garcia-Sáenz

jagsaenz@yahoo.com

L. Manso

luismansosanchez@gmail.com

M. Margelí

mmargeli@iconcologia.net

E. Dalmau

edalmau@tauli.cat

S. Pernas

spernas@iconcologia.net

A. Prat

alprat@clinic.ub.es

S. Servitja

sservitja@parcdesalutmar.cat

E. Ciruelos

eva.ciruelos@gmail.com

1 Department of Hematology and Medical Oncology, Hospital

G. Universitario Morales Meseguer, Avda. Marqués de los

Vélez, s/n, 30001 Murcia, Spain
2 Division of Medical Oncology, Hospital Clínico Universitario Lozano Blesa, Zaragoza, Spain

3 Department of Medical Oncology, Hospital Clínico Universitario San Carlos, Madrid, Spain

4 Department of Medical Oncology, University Hospital, 12 de Octubre, Madrid, Spain

5 Department of Medical Oncology, Breast Cancer Unit, B-ARGO Group, Institut Català d'Oncologia, Badalona, Spain

6 Department of Oncology, Parc Taulí Sabadell, Hospital Universitari, Barcelona, Spain

7 Department of Medical Oncology, Breast Cancer Unit, Institut Català d'Oncologia, Barcelona, Spain

8 Department of Medical Oncology, Hospital Clínic, Barcelona, Spain

9 Department of Medical Oncology, Hospital del Mar, Barcelona, Spain

10 Department of Medical Oncology, Breast Cancer Unit, University Hospital, 12 de Octubre, Madrid, Spain

11 HM Hospitales, Madrid, Spain 


\section{Abbreviations}

ET endocrine therapy

TAILORx Trial Assigning Individualized Options for Treatment

MINDACT Microarray in Node-Negative and 1 to 3 Positive lymph Node Disease may Avoid Chemotherapy EORTC 10041/BIG 3-04 study

DCIS ductal carcinoma in situ

\section{Introduction}

Breast cancer is a major public health problem due to its high incidence, prevalence, and mortality. It is by far the most common cancer among women in Spain (2012), accounting for $29 \%$ of all new cases of cancer in females. Moreover, it is the first cause of cancer-related mortality in the female population, accounting for $15.5 \%$ of female cancer deaths, and the 5-year prevalence is $40.8 \%$ [1].

Breast cancer is a heterogeneous disease with multiple intrinsic tumor subtypes [2]. Up to one in three patients will develop metastases depending on lymph node involvement and breast cancer subtype, despite remarkable progress in early diagnosis and treatment. A better understanding of prognostic and predictive factors is allowing individualization of treatment for early stage breast cancer patients. The aim of these guidelines is to summarize current evidence and to give evidence-based recommendations for clinical practice [3].

\section{Methodology}

These SEOM Guidelines have been developed with the consensus of ten breast cancer medical oncologists from the cooperative groups GEICAM (Spanish Breast Cancer
Research Group) and SOLTI (Spanish Collaborative Group for the Study, Treatment and Other Experimental Strategies in Solid Tumors). To assign a level of evidence and a grade of recommendation to the different statements of this treatment guideline, it was decided to use the Infectious Diseases Society of America-US Public Health Service Grading System for Ranking Recommendations in Clinical Guidelines to determine the quality of evidence and strength of recommendation in each of the consensus recommendations (Table 1). A full list of recommendations is provided in Table 2.

\section{Diagnosis and initial workup}

The following tests allow a correct diagnosis and prognosis approach to all patients in whom the presence of a breast tumor is suspected.

- Bilateral mammography and ultrasound of the breast and regional lymph nodes [I, A] [4]. Several new techniques such as 3D mammography or 3D ultrasound can increase diagnostic accuracy but are not routinely implemented.

- Core needle biopsy (preferably by ultrasound or stereotactic guidance). The study has to include the evaluation of the estrogen and progesterone receptor and HER2 gene expression [I, A]. Given the high inter-observer variability in the Ki-67 determination, it is important to be careful when using Ki67 to inform the decision-making process [5]. Fine needle aspiration or core biopsy of suspicious lymph nodes is recommended [II, A].

- MRI: is the most sensitive method for breast cancer staging but additional findings must be confirmed histologically due to the high false-positivity rate. Its use is not mandatory [I, B] and should be considered in cases of positive axillary nodes and occult primary breast cancer,
Table 1 Strength of recommendation and quality of evidence score

\begin{tabular}{ll}
\hline Category, grade & Definition \\
\hline Strength of recommendation & \\
A & Good evidence to support a recommendation for use \\
B & Moderate evidence to support a recommendation for use \\
C & Poor evidence to support a recommendation \\
D & Moderate evidence to support a recommendation against use \\
E & Good evidence to support a recommendation against use \\
Quality of evidence & \\
I & Evidence from $\geq 1$ properly randomized, controlled trial \\
II & Evidence from $\geq 1$ well-designed clinical trial, without rand- \\
& omization; from cohort or case-controlled analytic studies \\
& (preferably from $>1$ center); from multiple time series; or \\
& from dramatic results from uncontrolled experiments \\
III & Evidence from opinions of respected authorities, based \\
& on clinical experience, descriptive studies, or reports of \\
& expert committees \\
\hline
\end{tabular}


Table 2 SEOM clinical practice guidelines for early breast cancer (2018 update): summary of recommendations

Diagnosis and initial workup

Bilateral mammography and ultrasound of breast and regional lymph nodes in patients with suspected breast cancer

I, A

Core needle biopsy including evaluation of estrogen and progesterone receptors together with HER2 expression in patients with suspected breast cancer

Fine needle aspiration or core biopsy of suspicious lymph nodes

I, A

Bilateral breast MRI, with histologic confirmation of additional findings, as part of initial staging in patients with confirmed breast cancer, especially in patients with positive axillary nodes and occult primary breast cancer, Paget's disease of the nipple, lobular carcinoma and multifocal or multicentric lesions

Laboratory test as part of initial staging of patients with confirmed breast cancer

II, A

I, B

Additional staging with chest and abdomen CT and bone scan in patients with stage III disease and/or with clinical or laboratory suggesting metastases

Additional staging with PET-CT in patients with locally advanced breast cancer

III, B

Evaluation of cardiac function in patients requiring anthracyclines and/or trastuzumab

III, B

Surgery

Consideration of breast-conserving surgery as first surgical option in stages I-II

II, A

I, A

No indication of additional excision in patients with no ink on invasive tumor or DCIS after breast-conserving surgery

I, A

Contralateral mastectomy should be offered through an appropriate counseling process in BRCA1/2 mutation carriers

I, A

I, A

Sentinel lymph node (SLN) biopsy in patients with clinically negative axillary nodes

I, A

Omission of axillary lymph node dissection in patients with stage I-II disease and less than three positive axillary nodes after I, A

SLN biopsy and lumpectomy followed by adjuvant systemic therapy and radiotherapy

In patients with cNO tumors, additional treatment of axilla is not necessary if SLNB is negative after neoadjuvant chemotherapy

In patients with $\mathrm{cN}+$ tumors, if SLNB is negative after neoadjuvant chemotherapy (NAC):

ALND should be considered the standard procedure

I, A

ALND may be omitted in selected cases (pre-treatment marking of involved node, recovery of at least 2 SLNs, doubletracer technique)

Adjuvant radiotherapy

Hypofractionated schemes are preferred for external beam whole radiation therapy after breast-conserving surgery

In elderly patients, the benefit of radiotherapy after breast-conserving surgery for stage I tumors should be assessed individually

Nodal (supra-and infra-clavicular) irradiation should be administered in patients with four or more involved nodes after breast-conserving surgery

Nodal (supra-and infra-clavicular) irradiation is recommended in patients with one to three involved nodes after breastconserving surgery

In cN0 patients with pT1-2 tumors and positive SLNB, axillary irradiation may be used as an alternative to ALND or more involved nodes, $\mathrm{T} 4$ or T3N+tumors after mastectomy

Chest wall irradiation is recommended in patients with close or positive margins after mastectomy

Post-mastectomy irradiation should be considered in patients with T1-2 tumors and one to three involved nodes, or in patients with T3N0 tumors, after individually balancing risk factors for recurrence and patient preferences

Adjuvant radiotherapy is recommended after NAC in patients with stage III and T3NO disease, regardless of response to NAC, and in patients with node-positive disease after NACT

Adjuvant radiotherapy may be omitted after NAC in patients with stage II (excluding T3N0) who achieve a pCR and do not have additional risk factors for recurrence

Decision-making for systemic adjuvant treatment

OncotypeDX recurrence score (RS) may be used for prediction of the risk of distant recurrence at 9 years in patients with hormone receptor (HR)-positive and HER2-negative breast cancer treated with adjuvant endocrine therapy-only

OncotypeDX recurrence score (RS) may be used for prediction of the benefit of adjuvant chemotherapy in patients with hormone receptor (HR)-positive and HER2-negative

Prosigna risk of distant recurrence score (ROR) may be used for prediction of the risk of distant recurrence at 10 years in patients with HR-positive and HER2-negative breast cancer treated with adjuvant endocrine therapy-only for 5 years

Prosigna risk of distant recurrence score (ROR) may be used for prediction of the risk of late distant recurrence (years 5-10) in patients with HR-positive and HER2-negative breast cancer treated with adjuvant endocrine therapy-only for 5 years 
Table 2 (continued)

Mammaprint risk score may be used for prediction of the risk of distant recurrence at 5 years in patients with hormone recep- I, A tor (HR)-positive and HER2-negative breast cancer treated with adjuvant endocrine therapy-only

EndoPredict Epclin score may be used for prediction of the risk of distant recurrence at 10 years in patients with HR-positive I, B and HER2-negative breast cancer treated with adjuvant endocrine therapy-only for 5 years

EndoPredict Epclin score may be used for prediction of the risk of late distant recurrence (years 5-10) in patients with HR- I, B positive and HER2-negative breast cancer treated with adjuvant endocrine therapy-only for 5 years

Adjuvant and neoadjuvant systemic treatment of luminal breast cancer

Adjuvant endocrine therapy (ET) should be offered to any patient with HR-positive breast cancer

Adjuvant ET with tamoxifen for 5 years is recommended as a standard treatment for premenopausal women with HR-positive I, A breast cancer

Extended adjuvant ET with tamoxifen up to 10 years should be considered in high-risk patients

Adjuvant ET with exemestane plus ovarian function suppression should be offered to high-risk premenopausal breast cancer patients treated with adjuvant chemotherapy or in very young (under 35 years) women.

In high-risk patients not suitable or intolerant to aromatase inhibitors, tamoxifen plus ovarian function suppression may be considered as an alternative

Adjuvant ET for postmenopausal patients may consist in any of the following alternatives, after considering risk factors and individual preferences:

Aromatase inhibitor (AI) during 5 years

Tamoxifen for 2-3 years followed by an AI to complete 5 years

I, A

Tamoxifen for $2-3$ years followed by an AI during 5 years

II, B

Tamoxifen for 4.5-6 years followed by $2.5-5$ years of an AI

I, A

Adjuvant treatment with oral or intravenous bisphosphonates should be considered in postmenopausal patients with breast cancer who are candidates for adjuvant systemic therapy

Adjuvant chemotherapy with standard anthracyclines and/or taxane regimens for luminal breast cancer is recommended for tumors defined by either clinical or genomic risk factors: T2-4, axillary node involvement N2-3, extensive LVI, high Ki67, low ER expression, younger age and premenopausal status, and intermediate to high genomic score.

NAC is indicated in locally advanced hormone receptor (HR)-positive and HER2-negative breast cancer

A sequential regimen of anthracyclines and taxanes is recommended in those patients in which NAC is indicated for hormone receptor (HR)-positive and HER2-negative breast cancer

Aromatase inhibitors are preferred over tamoxifen in postmenopausal patients in which neoadjuvant endocrine therapy is indicated for hormone receptor (HR)-positive and HER2-negative breast cancer

Neoadjuvant endocrine therapy with AI plus ovarian suppression or with tamoxifen might be considered in selected premenopausal patients in which chemotherapy is not an option

Adjuvant and neoadjuvant systemic treatment of HER2 breast cancer

Addition of adjuvant trastuzumab to chemotherapy is recommended for HER2-positive breast cancer both in node-positive and in node-negative tumors with a tumor size $>1 \mathrm{~cm}$

12-month duration of trastuzumab should be considered standard

Addition of adjuvant trastuzumab to chemotherapy should be considered in most cases of node-negative HER2-positive breast cancer with tumor size of $0.5-1.0 \mathrm{~cm}$

For adjuvant chemotherapy of HER2-positive breast cancer, AC or EC for 4 cycles followed by 3 months of paclitaxel (P) or docetaxel $(\mathrm{D}$ or $\mathrm{T})$ both in combination with trastuzumab $(\mathrm{AC} / \mathrm{EC} \rightarrow \mathrm{P} / \mathrm{D}+\mathrm{H})$ or docetaxel, carboplatin and trastuzumab $(\mathrm{TCH})$ are the preferred regimens

In node-negative stage I tumors, an alternative less intense regimen with single-agent paclitaxel and trastuzumab for 12 weeks followed by single-agent trastuzumab (to complete a year) may be considered

Adjuvant dual HER2 blockade with trastuzumab and Pertuzumab for 18 cycles may be considered in patients with high-risk (node-positive and/or HR-negative) HER2-positive breast cancer. In patients that have received neoadjuvant treatment, Pertuzumab may be continued after surgery up to 18 cycles

Extended adjuvant treatment with neratinib after one year of trastuzumab may be considered in patients with node-positive and HR-positive HER2-positive breast cancer

Dual blockade with trastuzumab and pertuzumab and chemotherapy should be considered for the treatment of HER2-positive II, B breast cancer patients who meet criteria for neoadjuvant treatment ( $>2 \mathrm{~cm}$ tumor size and/or node-positive)

Adjuvant and neoadjuvant systemic treatment of triple-negative breast cancer

Adjuvant chemotherapy for triple-negative breast cancer should include an anthracycline and a taxane, although the regimen docetaxel-cyclophosphamide might be considered in patients with a high risk for cardiac toxicity 
Table 2 (continued)

\begin{tabular}{lll}
\hline Recommendation & Category, grade \\
\hline No adjuvant chemotherapy is recommended for node-negative pT1a triple-negative breast cancer & III, B \\
Adjuvant chemotherapy may be considered for 0.6-1 cm tumors after an adequate individualized balance & III, B \\
Neoadjuvant chemotherapy for triple-negative breast cancer should include sequential anthracyclines and taxanes & I, A \\
Carboplatin may be considered as part of neoadjuvant chemotherapy for triple-negative breast cancer patients & I, A \\
Adjuvant capecitabine for 6-8 cycles should be considered in high-risk triple-negative breast cancer with residual invasive & I, B \\
disease at surgery following standard neoadjuvant chemotherapy & & \\
Follow-up of early breast cancer & I, A \\
Breast cancer surveillance is recommended in order to detect breast cancer recurrence and second primary tumors, assess & II, A \\
physical and psychosocial long-term effects of breast cancer and its treatment, and promote a healthy lifestyle & III, A \\
Healthy lifestyles are recommended to prevent tumor recurrence & \\
Early breast cancer follow-up should include regular visits every 3-6 months in the first 2 years, every 6 months from years & \\
3-5 and annually thereafter & Annual ipsilateral (after breast-conserving surgery) and/or a contralateral mammography with ultrasound is recommended for & II, A \\
follow-up of early breast cancer &
\end{tabular}

Paget's disease of the nipple, lobular carcinoma and multifocal or multicentric lesions. It is recommended prior and after neoadjuvant treatment to define the extent of disease and monitor the response to treatment [III, A] [6].

- Additional studies: anamnesis with personal and family medical history and complete physical examination. Lab test (complete blood count, liver and renal function, alkaline phosphatase and calcium) are routinely used but do not seem to improve detection of occult metastatic disease [III, B] [7].

When disease is detected in stage III or when signs or symptoms or laboratory values suggest suspected metastasis, a more extensive study with thoracic CT, abdominal $\mathrm{CT}$ and bone scan [III, B] should be performed. PET/CT is also recommended for initial staging in locally advanced BC (LABC) [II, A] [8].

Evaluation of cardiac function is imperative when using anthracyclines or trastuzumab [I, A] [9].

\section{Principles of surgery}

Breast-conserving surgery is equivalent to mastectomy and must be considered as first option in most cases of stages I-II (I, A) [10]. No ink on invasive tumor or DCIS is considered an adequate margin after breast-conserving surgery [I, A] [11]. Mastectomy is still indicated in the following situations: tumor multicentricity, inability to achieve negative surgical margins after multiple resections, small breast size according to tumor volume, prior radiation therapy in the breast, or other contraindications for radiotherapy.
When mastectomy is performed, contralateral mastectomy as a prophylactic procedure is not indicated in most of the patients [12]. However, for carriers of BRCA1/2 mutations, the contralateral mastectomy should be offered through an appropriate risk-benefit assessment and counseling process [I, A] [13].

Sentinel lymph node biopsy (SLNB) is recommended for assessment of the involvement of axillary lymph nodes and should be performed in patients with clinically negative axillary nodes [I, A]. In patients with clinically positive axillary nodes, pathologic confirmation must be done by ultrasonography-guided fine needle aspiration (FNA) or core biopsy. Axillary lymph node dissection (ALND) is indicated in patients with positive SLN biopsy or confirmed preoperative pathologic axillary lymph node involvement. However, in patients with stage I-II disease and less than three positive axillary nodes after SLN biopsy and lumpectomy (and adjuvant radiotherapy indicated), ALND can be avoided without significant negative impact in DFS and OS when an adequate systemic postoperative treatment is provided [I, A] [14].

SLNB may be offered in patients with operable breast cancer before or after neoadjuvant chemotherapy (NACT). In patients with $\mathrm{cNO}$ axilla, additional axillary treatment is not necessary if SLNB is negative after NACT [I, A] [15]. In patients with $\mathrm{cN}+$ axilla who achieved ycN0 status after NACT, the ALND is recommended as standard procedure due to the high false-negative rate of the SLNB [I, A] [16]. In selected $\mathrm{cN}+$ cases, in which positive axillary node has been marked prior to NACT, the identification and recovery of $>2$ SLNs (including the marked node) with a doublecontrast technique (Tc99 and methylene blue) may avoid ALND [II, C]. 


\section{Recommendations of adjuvant radiotherapy}

Adjuvant radiotherapy (RT) should be performed in the case of:

- Breast-conserving surgery: external beam whole radiation therapy (WBRT). Hypofractionation schemes are preferred [I, A] [17]. If four or more nodes are involved, supra and infraclavicular radiotherapy is recommended [I, A]. In patients with one to three involved nodes after breast-conserving surgery, supra and infraclavicular nodal irradiation is recommended to minimize the risk of recurrence and potentially improve disease-specific survival [I, B] [18]. In addition, in patients with T1-2 tumors and $\mathrm{cN} 0$ and sentinel lymph node metastases, axillary irradiation is an alternative comparable to the ALND with less morbidity [I, B] [19].

- Mastectomy: chest wall and regional node irradiation including supraclavicular and internal mammary region is recommended in T4 tumors, node-positive T3 tumors, and if involvement of $\geq 4$ axillary lymph nodes [I, A]. In cases of close or positive margins, chest wall irradiation is also recommended [II, A] [18, 20]. T1-2 tumors with one to three involved nodes and T3NO tumors have an increased risk of locoregional recurrence after mastectomy. Post-mastectomy RT reduces the risk of recurrence and mortality and should be considered in these patients. However, benefit of adjuvant RT can be small in some subgroups of patients and must be discussed based on other risk factors such as grade, age, lymphovascular invasion, receptor status or lack of systemic therapy [I, B] [18, 21]. After neoadjuvant chemotherapy (NAC), irradiation is recommended for stage III disease, regardless of response to NAC, and for node-positive disease after NAC [I, A]. For patients presenting with stage II disease (excluding cT3N0) who achieve a pathologic complete response (pCR), radiotherapy could be omitted unless other risk factors [I, B]. [21-23].

Despite these recommendations, benefit of RT for stage I elderly patients has not been proved and should, therefore, be assessed individually [I, A] [24]. Some low-risk patients treated with conservative surgery could be spared whole breast RT and receive partial breast radiation or intraoperative treatment, although less evidence exists to support this approach [25].

\section{Principles of adjuvant systemic therapy. Genomic profiles in decision making in systemic adjuvant treatment}

\section{Principles of adjuvant systemic therapy}

Breast cancer (BC) is a heterogeneous disease, with different subtypes having a distinct biological, molecular, and clinical outcome. Systemic adjuvant treatment is commonly used in early breast cancer with the intention to reduce the rate of locoregional or systemic relapses and death derived from the disease. Treatment decisions are based on clinical (age, comorbidities) and pathologic factors (tumor size, nodal status, grade, hormone receptor (HR) status and HER2 status). Multigenic tests provide information beyond standard clinical and pathologic prognostic factors that can help in making treatment decisions.

\section{Prognostic gene expression-based assays}

Several prognostic gene expression-based assays have been developed to personalize the decision regarding the addition of chemotherapy (CT) in hormone receptor (HR)-positive and HER2-negative BC [26-30].

\section{Oncotype DX}

The test was initially validated as a prognostic biomarker in tumor samples from several prospective clinical trials (e.g., NSABP-B14 and TransATAC) [30-35]. A score cutpoint of less than 18 identified low-risk patients at 10 years that could be spared multi-agent chemotherapy, specially in node-negative disease. Less clear was the need of adjuvant chemotherapy in patients with a score of 18 to 31 . TAILORx prospective trial in HR+/HER2-/node-negative disease reported that in the intermediate risk group, defined as $11-25$, no benefit of adding chemotherapy to endocrine therapy was observed in the overall population at 9 years, although some benefit of chemotherapy was found in women $\leq 50$ years of age [29]. An important point is that the vast majority of patients (74\%) recruited in TAILORx were clinically low risk (i.e., $63 \%$ had tumor sizes $1-2 \mathrm{~cm}$ ). Indeed, no clear differences in survival outcomes were observed between the intermediate groups (with or without chemotherapy) compared to the low-risk group (i.e., $<11$ ). The RxPONDER prospective clinical trial in 1-3 positive nodes and $\mathrm{RS}<25$ will shed more light regarding the ability of OncotypeDX RS to predict chemotherapy benefit. Level of evidence: IA for the prediction of adjuvant chemotherapy benefit in patients with clinically low-risk disease and a recurrence score 11-25; IA for the prediction of the risk of distant recurrence at 9 years if treated with adjuvant endocrine therapy-only.

\section{Prosigna}

This assay classifies a tumor to one of the 4 intrinsic subtypes (Luminal A, Luminal B, HER2-enriched and basal-like) and provides a prognostic 10-year Risk of distant Recurrence (ROR) score [36], which integrates genomic data together 
with tumor size and nodal status (to determine the risk cutoffs). ROR has now been validated retrospectively in nodenegative and node-positive disease in tumor samples from several large adjuvant studies (i.e., TransATAC, ABCSG-08 and Danish cohort) [37-41]. In a recent head-to-head comparison with OncotypeDX RS and EndoPredict in 774 tumor samples from TransATAC, ROR provided a more accurate long-term prognostic information than OncotypeDX, similar to EndoPredict [41, 42]. Prospective validation of the ability of Prosigna to predict adjuvant chemotherapy survival benefit is currently ongoing in large phase III trial in UK (i.e., the OPTIMA trial), in patients $\geq 40$ years of age with HR+/HER 2-, $>3 \mathrm{~cm}$ or node-positive tumors and an ROR below 60 (i.e., low/intermediate). A recent published trial showed the prognostic and predictive value of Prosigna in premenopausal high-risk breast cancer patients treated with cyclophosphamide-based adjuvant chemotherapy [43]. Level of evidence: IB for the prediction of the risk of distant recurrence at 10 years if treated with 5 years of adjuvant endocrine therapy-only; Level of evidence: IB for the prediction of late distant recurrence (years 5-10) if treated with 5 years of adjuvant endocrine therapy-only.

\section{Mammaprint}

The prognostic value of Mammaprint was first validated in 295 patients with pT1 or pT 2 tumors with node-negative or node-positive disease [26, 27]. Since then, other retrospective validations of its prognostic value have been reported. Recently, the primary results of the prospective prognostic validation of Mammaprint have been reported. The MINDACT trial was an international phase III trial designed to evaluate the 5-year distant relapse-free survival (DRFS) in 748 patients, largely HR+/HER2-negative, with a genomic low-risk score and a clinically high-risk score (defined by a modified version of AdjuvantOnline!) when treated without adjuvant chemotherapy. The study met its primary endpoint and this group of patients had a 5-year DRFS of 94.7\% (92.5-96.2). However, longer follow-up is needed to determine if this group of patients continues to have an outstanding outcome after 5 years in order to spare them from adjuvant chemotherapy [44]. As a secondary underpowered analysis of the MINDACT trial, Mammaprint did not demonstrate the ability to predict chemotherapy survival benefit. Level of evidence: IA for the prediction of the risk of distant recurrence at 5 years if treated with adjuvant endocrine therapy-only.

\section{EndoPredict}

The prognostic value of EPclin has been validated retrospectively in tumors samples from 3 large phase III trials (ABCSG-6/ABCSG-8 [45] and TransATAC [46]), where patients did not receive any adjuvant chemotherapy. In addition, the assay has shown ability to predict late distant recurrence $[46,47]$. Level of evidence: IB for the prediction of the risk of distant recurrence at 10 years if treated with 5 years of adjuvant endocrine therapy-only. Level of evidence: IB for the prediction of late distant recurrence (years 5 to 10) if treated with 5 years of adjuvant endocrine therapy-only.

\section{Systemic treatment for luminal-type early stage breast cancer}

\section{Adjuvant endocrine therapy for early stage breast cancer}

There is robust evidence that endocrine therapy (ET) improves survival of early stage luminal breast cancer (BC). Adjuvant ET should be offered to any of these patients regardless of age, menopausal status, chemotherapy exposure, expression level of ER or PgR (if any or both are positive defined as ER and/or PR $\geq 1 \%$ ), and/or Her2 status [I, A]. There are several ET options. The individual choice would be adjusted to menopause status, comorbidity, and the risk of recurrence.

Tamoxifen is the most established adjuvant ET for both premenopausal and postmenopausal women as it reduces the risk of recurrence by $40 \%$ in all subgroups [48]. Two large trials (ATLAS and aTTom trial) concluded a higher benefit of continuing Tamoxifen until 10 years, which is recommended for high-risk tumors (at the cost of greater toxicity) [I, B] [49, 50].

Five years of tamoxifen remain the standard treatment for premenopausal women [I, A] [51], but other alternatives should be considered. Adjuvant exemestane plus ovarian function suppression as compared with tamoxifen plus ovarian function suppression or tamoxifen alone significantly reduced the likelihood of distant recurrence in highrisk adjuvant chemotherapy-treated premenopausal breast cancer patients [I, A]. After a median follow-up of 8 years, a survival improvement favoring ovarian suppression plus tamoxifen has been shown [52]. EBCTCG and SOFT trials also support adjuvant tamoxifen plus $\mathrm{GnRH}$ analogs as an alternative for high-risk patients not suitable to aromatase inhibitors [II, B] [53].

Several studies explored aromatase inhibitors (AIs) as an initial therapy [54-56], as sequential therapy following 2-3 years of tamoxifen [55-59] or as extended therapy [60, 61] for postmenopausal patients. Different options can be recommended: an AI for 5 years [I, A], tamoxifen for 2 to 3 years followed by an AI to complete 5 years [I, A] or during 5 years [II, B], or tamoxifen for 4.5 to 6 years followed by 2.5 to 5 years of an AI [I, A]. The recently published IDEAL trial did not find differences between 2.5 or 5 years 
of an AI after 5 years of any ET [62]. The option of 10 years of tamoxifen could be considered in patients with contraindication to AIs or who remain premenopausal.

There is strong evidence to consider the use of bisphosphonates (zoledronic acid $4 \mathrm{mg}$ intravenously every 6 months or clodronate $1.600 \mathrm{mg} / \mathrm{d}$ orally) but not an antiRANK-ligand antibody as additional adjuvant therapy for postmenopausal patients with breast cancer who are candidates for adjuvant systemic therapy. [I, A] [63].

\section{Adjuvant chemotherapy in hormone receptor-positive early BC}

The use of chemotherapy as adjuvant treatment for $\mathrm{ER}+\mathrm{Her} 2$-negative disease is recommended for high-risk tumors defined by either clinical or genomic profiling characteristics [I, A], considering: T2 to T4 tumors and/or axillary N2-3 involvement; extensive LVI, high KI67, low ER expression, younger age or premenopausal status; and intermediate to high genomic score. Standard anthracycline and taxane regimens are recommended [90] [I, A].

\section{Neoadjuvant chemotherapy for luminal breast cancer}

Neoadjuvant CT is indicated in LABC [I, A] to reduce the extent of surgery, to treat micrometastases promptly and to monitor the response.

All treatments recommended in the adjuvant setting may also be used in the preoperative setting. If chemotherapy is used, it should be delivered before surgery, without breaks, with the aim to increase the rate of breast-conserving surgery (pCR are infrequent in this setting). A sequential regimen of anthracyclines and taxanes is associated with increased probability of pCR and must be recommended [II, B] [64, 65]. In selected cases of postmenopausal patients with ER+/Her2- disease, neoadjuvant endocrine therapy (NET) during at least 16 weeks is a good option. The available data directly comparing neoadjuvant endocrine therapy (NET) with neoadjuvant chemotherapy are very limited. Some phase II trials and one meta-analysis showed similar response rates, but a significantly lower toxicity with NET [66]. AIs are better to tamoxifen as NET [I, A] [67]. The efficacy evaluation of NET has been performed according to surrogate parameters such as the decrease of the Ki67 levels or the preoperative endocrine prognostic index (PEPI) score [68]. Neoadjuvant ET in premenopausal patients is debatable; an AI with ovarian suppression or tamoxifen could be considered in selected cases in which chemotherapy is not an option [II, D].

In the neoadjuvant setting, chemotherapy is an accepted treatment for tumors with node metastases and for tumors greater than $2 \mathrm{~cm}$ who are candidates to mastectomy. We use clinical and pathologic parameters (HR status, grade, $\mathrm{Ki}-67)$ to select patients for neoadjuvant chemotherapy (NCT), though there is no agreement about the cutoff point of Ki-67 and about the accuracy of this marker to predict chemotherapy response. Different genetic signatures have been evaluated in core needle biopsy before neoadjuvant therapy, as good predictors of response to neoadjuvant therapy, especially PAM50 ROR score [69], although this approach is currently considered experimental.

\section{Systemic treatment for early stage her2-positive breast cancer}

\section{Adjuvant treatment for HER2-positive disease [70-77]}

The addition of trastuzumab to chemotherapy has dramatically improved prognosis for early stage HER2-positive breast cancer patients. The benefits of trastuzumab are independent of age, tumor size, nodal and HR status. Adjuvant trastuzumab is recommended in node-positive and node-negative tumors with a tumor size $>1 \mathrm{~cm}[\mathrm{I}, \mathrm{A}]$. No level I evidence exists regarding the use of trastuzumab in node-negative and tumors $\leq 1 \mathrm{~cm}$, although it might be considered in most patients with tumor size $0.5-1.0 \mathrm{~cm}$ [II, B]. While trastuzumab added sequentially after chemotherapy has demonstrated activity, results from the NCCTG N9831 study and a meta-analysis suggest better outcomes when trastuzumab is given concurrently with taxane-based treatment. Thus, AC or EC for 4 cycles followed by 3 months of paclitaxel $(\mathrm{P})$ or docetaxel (D or T) both in combination with trastuzumab $(\mathrm{AC} / \mathrm{EC} \rightarrow \mathrm{P} / \mathrm{D}+\mathrm{H})$ or docetaxel, carboplatin and trastuzumab $(\mathrm{TCH})$ are preferred regimens [I, A]. It is important to consider cardiac risk factors when determining the appropriate chemotherapy backbone to administer with trastuzumab. In small, node-negative tumors (stageI), although not all patients require adjuvant trastuzumabbased chemotherapy (particularly those with pT1aN0), a less intense chemotherapy regimen such as single-agent paclitaxel and trastuzumab for 12 weeks followed by singleagent trastuzumab to complete one year provides excellent outcomes [II, B]. To date, 12-month duration of trastuzumab remains the standard of care in most situations [I, A]. However, in patients with cardiac toxicity or at very high risk of cardiac toxicity, a shorter course (i.e., 6 months duration) can be considered. Trastuzumab may also be safely combined with either radiotherapy or endocrine therapy.

While there have been many efforts to improve outcomes even further, the addition of novel anti-HER2 therapies to trastuzumab, such as pertuzumab or neratinib, has led to significant, but modest improvements in DFS, and no impact on OS has yet been reported. Balancing risks and 
benefits is critical when evaluating adjuvant dual blockade with pertuzumab plus trastuzumab or extended HER2 inhibition with neratinib following 1-year of trastuzumab. Up to 18 cycles of adjuvant pertuzumab in combination with trastuzumab-based chemotherapy showed a $19 \%$ relative reduction in invasive disease-free survival (iDFS) at 3 years in the large phase III APHINITY trial. When stratification factors were evaluated, patients with highrisk features such as node positive or HR negative derived a significant iDFS, whereas patients with node negative or $\mathrm{HR}+$ disease did not. Although longer follow-up is needed, EMA states that the available data do not allow concluding for a positive benefit-risk ratio in the overall population. However, EMA considers that benefit is clearly shown in the high-risk population (HR-negative or node-positive) [I, B] for a total duration of 18 cycles of dual blockade, regardless if it was initiated in the adjuvant or the neoadjuvant setting.

The addition of 1 year of adjuvant neratinib improved iDFS in patients with HER2-positive early breast cancer after 1 year of trastuzumab, as demonstrated in the phase III EXTENET trial. However, the benefit was higher in patients with HR-positive and node-positive disease, at the expense of increased diarrhea [I, B]. Recently, neratinib has been approved by EMA, which restricted its use to $\mathrm{HR}+$ disease. However, there are no data on the added benefit of neratinib in patients who also received pertuzumab.

\section{Neoadjuvant treatment for HER2-positive disease [78-81]}

In the neoadjuvant setting, trastuzumab in combination with chemotherapy (taxane and anthracycline based) has been the standard treatment for HER2-positive tumors with nodal involvement or tumor size $>2 \mathrm{~cm}$. Two phase II trials evaluated the addition of pertuzumab to trastuzumab-based chemotherapy, demonstrating higher rates of pCR. These data, along with its efficacy in terms of OS in the metastatic setting, led to the accelerated approval of this combination in the neoadjuvant scenario. Dual blockade with trastuzumab and pertuzumab and chemotherapy (anthracycline plus taxane based, or anthracycline-free regimens) should be considered for the treatment of HER2-positive breast cancer patients who meet criteria for neoadjuvant treatment (i.e., $>2 \mathrm{~cm}$ tumor size or node positive). According to EMA and FDA, treatment with dual HER2 blockade can be continued after surgery for up to 18 cycles [I, B]. At this point, the type of pathological response at surgery should not guide the duration of trastuzumab or pertuzumab since there are no data supporting one strategy or another. Finally, the addition of lapatinib to trastuzumab and chemotherapy has not consistently improved pCR rates and long-term outcome. To date, this combination cannot be recommended for the treatment of patients with early stage HER2-positive breast cancer [I,E].

\section{Systemic therapy for early stage triple-negative breast cancer}

\section{Adjuvant treatment for triple-negative disease}

Triple-negative breast cancer (TNBC) is a heterogeneous disease comprising approximately $15 \%$ of all breast cancers. With the exception of medullary, adenoid cystic, and apocrine carcinomas that have a better outcome, TNBCs have generally an aggressive behavior.

Conventional chemotherapy remains the mainstay of adjuvant systemic treatment for most patients with early TNBC. Adjuvant chemotherapy should include an anthracycline and a taxane [I, B], although the regimen docetaxelcyclophosphamide might be considered in patients with a high risk for cardiac toxicity. Nevertheless, women with $\mathrm{T} 1 \mathrm{a} / \mathrm{bN} 0$ tumors have an excellent prognosis without chemotherapy [82]. No adjuvant chemotherapy is recommended in tumors equal or less than $0.5 \mathrm{~cm}(\mathrm{pT} 1 \mathrm{a})$, and for $0.6-1 \mathrm{~cm}$ tumors, it has to be discussed and balanced [III, B]. No robust, prospective randomised data exist on the use of platinum compounds in the adjuvant setting, either in unselected triple-negative tumors or BRCA 1/2 mutation carriers.

\section{Neoadjuvant treatment for triple-negative disease}

Neoadjuvant therapy in TNBC leads to pCR rates of $30-40 \%$, which has been associated with an excellent prognosis [65]. It is recommended that a sequential regimen of anthracyclines and taxanes is used for the vast majority of patients [I, B]. An improved pCR was observed with Nabpaclitaxel compared to solvent-based weekly paclitaxel (43 vs $34 \%$ ) in a head to head phase III neoadjuvant trial. This effect was seen in all subgroups, especially in TNBC patients [83].

Platinum-based neoadjuvant chemotherapy significantly increased pCR from $37.0 \%$ to $52.1 \%$ (OR $1.96,95 \%$ CI $1.46-2.62, P<0.001$ ), with significant higher risk of grade 3 and 4 hematological AEs [I, A]. In the 96 BRCA-mutated patients included in two randomized controlled trials, the addition of carboplatin was not associated with significantly increased pCR rate [84]. The effect of those compounds on long-term outcomes is unknown [I, B].

Addition of 6-8 cycles of adjuvant capecitabine therapy among patients who had residual invasive disease on pathological testing after anthracycline and taxane-based neoadjuvant chemotherapy was safe and effective in prolonging DFS and OS in the randomized phase III CREATE-X trial [85]. Based on those results and a meta-analysis [86], adjuvant 
capecitabine can be considered in high-risk TNBC patients with residual invasive disease at surgery following standard neoadjuvant chemotherapy [I, B] This treatment, though, must be balanced against potential toxicities.

Recommendation: sequential regimens of anthracyclines followed by taxanes are the standard treatment. Platinumbased neoadjuvant chemotherapy may be considered an option in TNBC patients [I, A].

\section{Follow-up}

Follow-up after surgery and adjuvant treatment of breast cancer patients is widely reported in guidelines worldwide. Surveillance for breast cancer recurrence, screening for second primary cancers, assessment and management of physical and psychosocial long-term and late effects of breast cancer and its treatment, and health promotion are encouragingly recommended [I, A] [87]. Healthy lifestyles, such as physical exercise and avoidance of obesity, are recommended to prevent tumor recurrence [II, A] [88].

Despite the fact that no randomized data exist to support any particular follow-up sequence or protocol, balancing patient needs and follow-up costs, we recommend regular visits every 3-6 months in the first 2 years, every 6 months from years 3-5 and annually thereafter [III, A]. Every visit should include a thorough history, eliciting of symptoms and a physical examination. Annual ipsilateral (after BCT) and/ or a contralateral mammography with ultrasound is recommended [II, A]. An MRI of the breast may be indicated for young patients, especially in cases of dense breast tissue and genetic or familial predispositions [89].

\section{Compliance with ethical standards}

Conflict of interest FAP reports grants from Roche and Celgene, other from Roche, Celgene, Eisai, AstraZeneca, Pfizer, Pierre Fabre and Novartis, outside the submitted work. RA has nothing to disclose. JAGS reports personal fees from Novartis and Celgene, grants from AstraZeneca, other from Roche, outside the submitted work. LM reports other from Roche, other from AstraZeneca, other from Novartis, other from Tesaro, outside the submitted work. MM has received speaker honoraria and funding for some independent medical education activities from Roche, Novartis, Astra Zeneca, Kern and Merck outside the submitted work. ED has nothing to disclose. SP reports personal fees and non-financial support from Roche, other from Polyphor, nonfinancial support from Pierre Fabre, outside the submitted work. AP reports personal fees from Nanostring, Roche, Amgen, Novartis, Eli Lilly and Pfizer, and grants from Roche, Novartis and Nanostring, all outside the submitted work. AP is part of the scientific advisory board of Oncolytics Biotech. SS has nothing to disclose. EC reports personal fees and other from Roche, Pfizer, Novartis, MSD, Celgene, Lilly and Pierre Fabre, outside the submitted work.

Ethical standards The current study has been performed in accordance with the ethical standards laid down in the 1964 Declaration of Hel- sinki and its later amendments. This article does not contain any studies with human participants or animals performed by any of the authors.

Informed consent For this type of study, no informed consent is required.

Open Access This article is distributed under the terms of the Creative Commons Attribution 4.0 International License (http://creativeco mmons.org/licenses/by/4.0/), which permits unrestricted use, distribution, and reproduction in any medium, provided you give appropriate credit to the original author(s) and the source, provide a link to the Creative Commons license, and indicate if changes were made.

\section{References}

1. SEOM: Las Cifras Del Cáncer En España. 2018. https://seom. org/seomcms/images/stories/recursos/Las_Cifras_del_cance r_en_Espana2018.pdf. Accessed 15 Oct 2018.

2. Cho S-H, Jeon J, Kim SI. Personalized medicine in breast cancer: a systematic review. J Breast Cancer. 2012;15:265-72.

3. Peto R, Davies C, Godwin J, Gray R, Pan HC, Clarke M, et al. Comparisons between different polychemotherapy regimens for early breast cancer: meta-analyses of long-term outcome among 100,000 women in 123 randomised trials. Lancet. 2012;379:432-44.

4. Perry N, Broeders M, de Wolf C, Tornberg S, Holland R, von Karsa L. European guidelines for quality assurance in breast cancer screening and diagnosis. Fourth edition-summary document. Ann Oncol. 2007;19:614-22.

5. Polley YC, Leung SCY, McShane LM, Gao D, Hugh JC, Mastropasqua MG, et al. International Ki67 in Breast Cancer Working Group of the Breast International Group and North American Breast Cancer Group. An International Ki67 Reproducibility Study. J Natl Cancer Inst. 2013;105:1897-906.

6. Sardanelli F, Boetes C, Borisch B, Decker T, Federico M, Gilbert FJ, et al. Magnetic resonance imaging of the breast: recommendations from the EUSOMA working group. Eur J Cancer. 2010;46:1296-316.

7. Louie RJ, Tonneson JE, Gowarty M, Goodney PP, Barth RJ, Rosenkranz KM. Complete blood counts, liver function tests, and chest $\mathrm{x}$-rays as routine screening in early-stage breast cancer: value added or just cost? Breast Cancer Res Treat. 2015;154:99-103.

8. Caresia Aroztegui AP, García Vicente AM, Alvarez Ruiz S, Delgado Bolton RC, Orcajo Rincon J, Garcia Garzon JR, et al. Oncology Task Force of the Spanish Society of Nuclear Medicine and Molecular Imaging: 18F-FDG PET/CT in breast cancer: evidence-based recommendations in initial staging. Tumour Biol. 2017;39:1010428317728285.

9. López-Fernández T, Martín García A, Santaballa Beltrán A, Montero Luis Á, García Sanz R, Mazón Ramos P, et al. Cardio-Onco-Hematología en la práctica clínica. Documento de consenso y recomendaciones. Rev Española Cardiol. 2017;70:474-86.

10. Association of Breast Surgery at Baso 2009. Surgical guidelines for the management of breast cancer. Eur J Surg Oncol. 2009;35(Suppl 1):1-22.

11. Buchholz TA, Somerfield MR, Griggs JJ, El-Eid S, Hammond $\mathrm{MEH}, \mathrm{Lyman} \mathrm{GH}$, et al. Margins for breast-conserving surgery with whole-breast irradiation in Stage I and II invasive breast cancer: American Society of Clinical Oncology Endorsement of the Society of Surgical Oncology/American Society for Radiation Oncology Consensus Guideline. J Clin Oncol. 2014;32:1502-6. 
12. Fayanju OM, Stoll CRT, Fowler S, Colditz GA, Margenthaler JA. Contralateral prophylactic mastectomy after unilateral breast cancer. Ann Surg. 2014;260:1000-10.

13. Li X, You R, Wang X, Liu C, Xu Z, Zhou J, et al. Effectiveness of prophylactic surgeries in BRCA1 or BRCA2 mutation carriers: a meta-analysis and systematic review. Clin Cancer Res. 2016;22:3971-81.

14. Giuliano AE, McCall L, Beitsch P, Whitworth PW, Blumencranz P, Leitch AM, et al. Locoregional recurrence after sentinel lymph node dissection with or without axillary dissection in patients with sentinel lymph node metastases. Trans Meet Am Surg Assoc. 2010;128:12-21.

15. van der Heiden-van der Loo M, de Munck L, Sonke GS, van Dalen T, van Diest PJ, van den Bongard HJGD, et al. Population based study on sentinel node biopsy before or after neoadjuvant chemotherapy in clinically node negative breast cancer patients: identification rate and influence on axillary treatment. Eur J Cancer. 2015;51:915-21.

16. van Nijnatten TJA, Schipper RJ, Lobbes MBI, Nelemans PJ, Beets-Tan RGH, Smidt ML. The diagnostic performance of sentinel lymph node biopsy in pathologically confirmed node positive breast cancer patients after neoadjuvant systemic therapy: a systematic review and meta-analysis. Eur J Surg Oncol. 2015;41:1278-87.

17. Whelan TJ, Pignol J-P, Levine MN, Julian JA, MacKenzie R, Parpia S, et al. Long-term results of hypofractionated radiation therapy for breast cancer. N Engl J Med. 2010;362:513-20.

18. EBCTCG (Early Breast Cancer Trialists' Collaborative Group), McGale P, Taylor C, Correa C, Cutter D, Duane F, et al. Effect of radiotherapy after mastectomy and axillary surgery on 10-year recurrence and 20-year breast cancer mortality: meta-analysis of individual patient data for 8135 women in 22 randomised trials. Lancet. 2014;83:212-35.

19. Donker M, van Tienhoven G, Straver ME, Meijnen P, van de Velde $\mathrm{CJH}$, Mansel RE, et al. Radiotherapy or surgery of the axilla after a positive sentinel node in breast cancer (EORTC 10981-22023 AMAROS): a randomised, multicentre, open-label, phase 3 noninferiority trial. Lancet Oncol. 2014;15:1303-10.

20. Jagsi R, Raad RA, Goldberg S, Sullivan T, Michaelson J, Powell $\mathrm{SN}$, et al. Locoregional recurrence rates and prognostic factors for failure in node-negative patients treated with mastectomy: implications for postmastectomy radiation. Int J Radiat Oncol. 2005;62:1035-9.

21. McBride A, Allen P, Woodward W, Kim M, Kuerer HM, Drinka $\mathrm{EK}$, et al. Locoregional recurrence risk for patients With T1,2 breast cancer with 1-3 positive lymph nodes treated with mastectomy and systemic treatment. Int J Radiat Oncol. 2014;89:392-8.

22. Huang EH, Tucker SL, Strom EA, McNeese MD, Kuerer HM, Buzdar AU, et al. Postmastectomy radiation improves localregional control and survival for selected patients with locally advanced breast cancer treated with neoadjuvant chemotherapy and mastectomy. J Clin Oncol. 2004;22:4691-9.

23. Nagar H, Mittendorf EA, Strom EA, Perkins GH, Oh JL, Tereffe $\mathrm{W}$, et al. Local-regional recurrence with and without radiation therapy after neoadjuvant chemotherapy and mastectomy for clinically staged T3N0 breast cancer. Int J Radiat Oncol. 2011;81:782-7.

24. Hughes KS, Schnaper LA, Bellon JR, Cirrincione CT, Berry DA, McCormick B, et al. Lumpectomy plus tamoxifen with or without irradiation in women age 70 years or older with early breast cancer: long-term follow-up of CALGB 9343. J Clin Oncol. 2013;31:2382-7.

25. Correa C, Harris EE, Leonardi MC, Smith BD, Taghian AG, Thompson AM, et al. Accelerated partial breast irradiation: executive summary for the update of an ASTRO evidence-based consensus statement. Pract Radiat Oncol. 2016;7:73-9.
26. Paik S, Shak S, Tang G, Kim C, Baker J, Cronin M, et al. A multigene assay to predict recurrence of tamoxifen-treated, nodenegative breast cancer. N Engl J Med. 2004;351:2817-26.

27. Paik S, Tang G, Shak S, Kim C, Baker J, Kim W, et al. Gene expression and benefit of chemotherapy in women with nodenegative, estrogen receptor-positive breast cancer. J Clin Oncol. 2006;24:3726-34.

28. Sparano JA, Gray RJ, Makower DF, Pritchard KI, Albain KS, Hayes DF, et al. Prospective validation of a 21-gene expression assay in breast cancer. N Engl J Med. 2015;373:2005-14.

29. Sparano JA, Gray RJ, Makower DF, Pritchard KI, Albain KS, Hayes DF, et al. Adjuvant chemotherapy guided by a 21-gene expression assay in breast cancer. N Engl J Med. 2018;379:111-21.

30. Albain KS, Barlow WE, Shak S, Hortobagyi GN, Livingston RB, Yeh I-T, et al. Prognostic and predictive value of the 21-gene recurrence score assay in postmenopausal women with nodepositive, oestrogen-receptor-positive breast cancer on chemotherapy: a retrospective analysis of a randomised trial. Lancet Oncol. 2010;11:55-65.

31. Dowsett M, Cuzick J, Wale C, Forbes J, Mallon EA, Salter J, et al. Prediction of risk of distant recurrence using the 21-gene recurrence score in node-negative and node-positive postmenopausal patients with breast cancer treated with anastrozole or tamoxifen: a TransATAC study. J Clin Oncol. 2010;28:1829-34.

32. Mamounas EP, Tang G, Paik S, Bachner FL, Liu Q, Jeong J-H, et al: Prognostic impact of the 21-gene recurrence score (RS) on disease-free and overal survival of node-positive, ER positive breast cancer patients treates with adjuvant chemotherapy: results from NSABP B-28. J Clin Oncol. 2012,30:(27 suppl 1-1).

33. Solin LJ, Gray R, Goldstein LJ, Recht A, Baehner FL, Shak S, et al. Prognostic value of biologic subtype and the 21-gene recurrence score relative to local recurrence after breast conservation treatment with radiation for early stage breast carcinoma: results from the Eastern Cooperative Oncology Group E2197 study. Breast Cancer Res Treat. 2012;134:683-92.

34. Roberts MC, Miller DP, Shak S, Petkov VI. Breast cancer-specific survival in patients with lymph node-positive hormone receptorpositive invasive breast cancer and oncotype DX recurrence score results in the SEER database. Breast Cancer Res Treat. 2017;163:303-10.

35. Gluz O, Nitz UA, Christgen M, Kates RE, Shak S, Clemens M, et al. West German Study Group Phase III PlanB Trial: first prospective outcome data for the 21-gene recurrence score assay and concordance of prognostic markers by central and local pathology assessment. J Clin Oncol. 2016;34:2341-9.

36. Parker JS, Mullins M, Cheang MCU, Leung S, Voduc D, Vickery $\mathrm{T}$, et al. Supervised risk predictor of breast cancer based on intrinsic subtypes. J Clin Oncol. 2009;27:1160-7.

37. Nielsen TO, Parker JS, Leung S, Voduc D, Ebbert M, Vickery T, et al. A comparison of PAM50 intrinsic subtyping with immunohistochemistry and clinical prognostic factors in tamoxifentreated estrogen receptor-positive breast cancer. Clin Cancer Res. 2010;16:5222-32.

38. Gnant M, Filipits M, Greil R, Stoeger H, Rudas M, Bago-Horvath $\mathrm{Z}$, et al. Predicting distant recurrence in receptor-positive breast cancer patients with limited clinicopathological risk: using the PAM50 Risk of Recurrence score in 1478 postmenopausal patients of the ABCSG- 8 trial treated with adjuvant endocrine therapy alone. Ann Oncol. 2014;25:339-45.

39. Gnant M, Sestak I, Filipits M, Dowsett M, Balic M, LopezKnowles E, et al. Identifying clinically relevant prognostic subgroups of postmenopausal women with node-positive hormone receptor-positive early-stage breast cancer treated with endocrine therapy: a combined analysis of ABCSG- 8 and ATAC using the 
PAM50 risk of recurrence score and intrinsic subtype. Ann. 2015;26:1685-91.

40. Dowsett M, Sestak I, Lopez-Knowles E, Sidhu K, Dunbier AK, Cowens JW, et al. Comparison of PAM50 risk of recurrence score with oncotype DX and IHC4 for predicting risk of distant recurrence after endocrine therapy. J Clin Oncol. 2013;31:2783-90.

41. Lænkholm A-V, Jensen M-B, Eriksen JO, Rasmussen BB, Knoop AS, Buckingham W, et al. PAM50 risk of recurrence score predicts 10-year distant Recurrence in a comprehensive danish cohort of postmenopausal women allocated to 5 years of endocrine therapy for hormone receptor-positive early breast cancer. J Clin Oncol. 2018;36:735-40.

42. Sestak I, Buus R, Cuzick J, Dubsky P, Kronenwett R, Denkert C, et al. Comparison of the performance of 6 prognostic signatures for estrogen receptor-positive breast cancer: a secondary analysis of a randomized clinical trial. JAMA Oncol. 2018;4:545-53.

43. Jensen M-B, Lænkholm A-V, Nielsen TO, Eriksen JO, Wehn P, Hood T, et al. The Prosigna gene expression assay and responsiveness to adjuvant cyclophosphamide-based chemotherapy in premenopausal high-risk patients with breast cancer. Breast Cancer Res. 2018;20:79.

44. Cardoso F, van't Veer LJ, Veer LJ, Bogaerts J, Slaets L, Viale $\mathrm{G}$, Delaloge S, et al. 70-gene signature as an aid to treatment decisions in early-stage breast cancer. N Engl J Med. 2016;375:717-29.

45. Filipits M, Rudas M, Jakesz R, Dubsky P, Fitzal F, Singer CF, et al. A new molecular predictor of distant recurrence in ERpositive, HER2-negative breast cancer adds independent information to conventional clinical risk factors. Clin Cancer Res. 2011;17:6012-20.

46. Buus R, Sestak I, Kronenwett R, Denkert C, Dubsky P, Krappmann K, et al. Comparison of EndoPredict and EPclin with Oncotype DX recurrence score for prediction of risk of distant recurrence after endocrine therapy. J Natl Cancer Inst. 2016. https ://doi.org/10.1093/jnci/djw149.

47. Dubsky P, Brase JC, Jakesz R, Rudas M, Singer CF, Greil R, et al. The EndoPredict score provides prognostic information on late distant metastases in ER+/HER2- breast cancer patients. Br J Cancer. 2013;109:2959-64.

48. Early Breast Cancer Trialists' Collaborative Group (EBCTCG). Effects of chemotherapy and hormonal therapy for early breast cancer on recurrence and 15-year survival: an overview of the randomised trials. Lancet. 2005;365:1687-717.

49. Davies C, Pan H, Godwin J, Gray R, Arriagada R, Raina V, et al. Long-term effects of continuing adjuvant tamoxifen to 10 years versus stopping at 5 years after diagnosis of oestrogen receptor-positive breast cancer: aTLAS, a randomised trial. Lancet. 2013;381:805-16.

50. Gray R, Rea D, Handley D, Bowden S, Perry P. aTTom: long-term effects of continuing adjuvant tamoxifen to 10 years versus stopping at 5 years in 6.953 women with early breast cancer. J Clin Oncol. 2013;31s:a:5.

51. Early Breast Cancer Trialists' Collaborative Group (EBCTCG), Davies C, Godwin J, Gray R, Clarke M, Cutter D, et al. Relevance of breast cancer hormone receptors and other factors to the efficacy of adjuvant tamoxifen: patient-level meta-analysis of randomised trials. Lancet. 2011;378:771-84.

52. Francis PA, Pagani O, Fleming GF, Walley BA, Colleoni M, Láng I, et al. Tailoring adjuvant endocrine therapy for premenopausal breast cancer. N Engl J Med. 2018;379:122-37.

53. LHRH-agonists in Early Breast Cancer Overview group, Cuzick J, Ambroisine L, Davidson N, Jakesz R, Kaufmann M, et al. Use of luteinising-hormone-releasing hormone agonists as adjuvant treatment in premenopausal patients with hormone-receptor-positive breast cancer: a meta-analysis of individual patient data from randomised adjuvant trials. Lancet. 2007;369:1711-23.
54. Howell A, Cuzick J, Baum M, Buzdar A, Dowsett M, Forbes JF, et al. Results of the ATAC (arimidex, tamoxifen, alone or in combination) trial after completion of 5 years' adjuvant treatment for breast cancer. Lancet. 2005;365:60-2.

55. BIG 1-98 Collaborative Group, Mouridsen H, Giobbie-Hurder A, Goldhirsch A, Thürlimann B, Paridaens R, et al. Letrozole therapy alone or in sequence with tamoxifen in women with breast cancer. N Engl J Med. 2009;361:766-76.

56. Derks MGM, Blok EJ, Seynaeve C, Nortier JWR, Kranenbarg EM-K, Liefers G-J, et al. Adjuvant tamoxifen and exemestane in women with postmenopausal early breast cancer (TEAM): 10-year follow-up of a multicentre, open-label, randomised, phase 3 trial. Lancet Oncol. 2017;18:1211-20.

57. Boccardo F, Rubagotti A, Puntoni M, Guglielmini P, Amoroso D, Fini A, et al. Switching to anastrozole versus continued tamoxifen treatment of early breast cancer: preliminary results of the Italian Tamoxifen Anastrozole Trial. J Clin Oncol. 2005;23:5138-47.

58. Coombes RC, Hall E, Gibson LJ, Paridaens R, Jassem J, Delozier $\mathrm{T}$, et al. A randomized trial of exemestane after two to three years of tamoxifen therapy in postmenopausal women with primary breast cancer. N Engl J Med. 2004;350:1081-92.

59. Jakesz R, Jonat W, Gnant M, Mittlboeck M, Greil R, Tausch $\mathrm{C}$, et al. Switching of postmenopausal women with endocrineresponsive early breast cancer to anastrozole after 2 years' adjuvant tamoxifen: combined results of ABCSG trial 8 and ARNO 95 trial. Lancet. 2005;366:455-62.

60. Goss PE, Ingle JN, Pater JL, Martino S, Robert NJ, Muss HB, et al. Late extended adjuvant treatment with letrozole improves outcome in women with early-stage breast cancer who complete 5 years of tamoxifen. J Clin Oncol. 2008;26:1948-55.

61. Jakesz R, Greil R, Gnant M, Schmid M, Kwasny W, Kubista E, et al. Extended adjuvant therapy with anastrozole among postmenopausal breast cancer patients: results from the randomized Austrian Breast and Colorectal Cancer Study Group Trial 6a. J Natl Cancer Inst. 2007;99:1845-53.

62. Blok EJ, Kroep JR, Meershoek-Klein Kranenbarg E, Duijm-de Carpentier M, Putter H, van den Bosch J, et al. Optimal duration of extended adjuvant endocrine therapy for early breast cancer; results of the IDEAL trial (BOOG 2006-05). J Natl Cancer Inst. 2018;110:40-8.

63. Dhesy-Thind S, Fletcher GG, Blanchette PS, Clemons MJ, Dillmon MS, Frank ES, et al. Use of adjuvant bisphosphonates and other bone-modifying agents in breast cancer: a Cancer Care Ontario and American Society of Clinical Oncology Clinical Practice Guideline. J Clin Oncol. 2017;35:2062-81.

64. von Minckwitz G, Untch M, Nüesch E, Loibl S, Kaufmann M, Kümmel S, et al. Impact of treatment characteristics on response of different breast cancer phenotypes: pooled analysis of the German neo-adjuvant chemotherapy trials. Breast Cancer Res Treat. 2011;125:145-56.

65. Cortazar P, Zhang L, Untch M, Mehta K, Costantino JP, Wolmark N, et al. Pathological complete response and long-term clinical benefit in breast cancer: the $\mathrm{CTNeoBC}$ pooled analysis. Lancet. 2014:384:164-72.

66. Alba E, Calvo L, Albanell J, De la Haba JR, Arcusa Lanza A, Chacon JI, et al. Chemotherapy (CT) and hormonotherapy (HT) as neoadjuvant treatment in luminal breast cancer patients: results from the GEICAM/2006-03, a multicenter, randomized, phase-II study. Ann Oncol. 2012;23:3069-74.

67. Smith IE, Dowsett M, Ebbs SR, Dixon JM, Skene A, Blohmer J-U, et al. Neoadjuvant treatment of postmenopausal breast cancer with anastrozole, tamoxifen, or both in combination: the immediate preoperative anastrozole, tamoxifen, or combined with tamoxifen (IMPACT) multicenter double-blind randomized trial. J Clin Oncol. 2005;23:5108-16. 
68. Ellis MJ, Suman VJ, Hoog J, Lin L, Snider J, Prat A, et al. Randomized phase II neoadjuvant comparison between letrozole, anastrozole, and exemestane for postmenopausal women with estrogen receptor-rich stage 2 to 3 breast cancer: clinical and biomarker outcomes and predictive value of the baseline PAM50-based intrinsic subtype-ACOSOG Z1031. J Clin Oncol. 2011;29:2342-9.

69. Prat A, Galvan P, Jimenez B, Buckingham W, Jeiranian HA, Schaper C, et al. Prediction of response to neoadjuvant chemotherapy using core needle biopsy samples with the Prosigna assay. Clin Cancer Res. 2016;22:560-6.

70. Moja L, Tagliabue L, Balduzzi S, Parmelli E, Pistotti V, Guarneri V, et al. Trastuzumab containing regimens for early breast cancer. Cochrane Database Syst Rev. 2012:CD006243.

71. Perez EA, Romond EH, Suman VJ, Jeong J-H, Sledge G, Geyer CE, et al. Trastuzumab plus adjuvant chemotherapy for human epidermal growth factor receptor 2-positive breast cancer: planned joint analysis of overall survival from NSABP B-31 and NCCTG N9831. J Clin Oncol. 2014;32:3744-52.

72. Cameron D, Piccart-Gebhart MJ, Gelber RD, Procter M, Goldhirsch A, de Azambuja E, et al. 11 years' follow-up of trastuzumab after adjuvant chemotherapy in HER2-positive early breast cancer: final analysis of the HERceptin Adjuvant (HERA) trial. Lancet. 2017;389:1195-205.

73. Slamon D, Eiermann W, Robert N, Giermek J, Martín M, Jasiówka M, et al. Ten year follow-up of the BCIRG-006 trial comparing doxorubicin plus cyclophosphamide followed by docetaxel (AC-T) with doxorubicin plus cyclophosphamide followed by docetaxel and trastuzumab (AC-TH) with docetaxel. Cancer Res. 2015;76(4_suppl):S5-04.

74. Tolaney SM, Barry WT, Guo H, Dillon D, Dang CH-T, Yardley DA, et al. Seven-year follow-up of adjuvant paclitaxel $(T)$ and trastuzumab (H) (APT trial) for node-negative, HER2-positive breast cancer. J Clin Oncol. 2017;15_suppl(15):511.

75. Chan A, Delaloge S, Holmes FA, Moy B, Iwata H, Harvey VJ, et al. Neratinib after trastuzumab-based adjuvant therapy in patients with HER2-positive breast cancer (ExteNET): a multicentre, randomised, double-blind, placebo-controlled, phase 3 trial. Lancet Oncol. 2016;17:367-77.

76. von Minckwitz G, Procter M, de Azambuja E, Zardavas D, Benyunes M, Viale G, et al. Adjuvant pertuzumab and trastuzumab in early HER2-positive breast cancer. N Engl J Med. 2017;377:122-31.

77. Earl H, Hiller L, Vallier A, Loi S, Howe D, Higgins H. PERSEPHONE: 6 versus 12 months (m) of adjuvant trastuzumab in patients (pts) with HER2 positive (+) early breast cancer (EBC): Randomised phase 3 non-inferiority trial with definitive 4-year (yr) disease-free survival (DFS) results. J Clin Oncol. 2018;36:506

78. Gianni L, Eiermann W, Semiglazov V, Manikhas A, Lluch A, Tjulandin S, et al. Neoadjuvant chemotherapy with trastuzumab followed by adjuvant trastuzumab versus neoadjuvant chemotherapy alone, in patients with HER2-positive locally advanced breast cancer (the NOAH trial): a randomised controlled superiority trial with a parallel HER2-negative cohort. Lancet. 2010;375:377-84.

79. Gianni L, Pienkowski T, Im Y-H, Roman L, Tseng L-M, Liu $\mathrm{M}-\mathrm{C}$, et al. Efficacy and safety of neoadjuvant pertuzumab and trastuzumab in women with locally advanced, inflammatory, or early HER2-positive breast cancer (NeoSphere): a randomised multicentre, open-label, phase 2 trial. Lancet Oncol. 2012;13:25-32.

80. Schneeweiss A, Chia S, Hickish T, Harvey V, Eniu A, Hegg R, et al. Pertuzumab plus trastuzumab in combination with standard neoadjuvant anthracycline-containing and anthracycline-free chemotherapy regimens in patients with HER2-positive early breast cancer: a randomized phase II cardiac safety study (TRYPHAENA). Ann Oncol. 2013;24:2278-84.

81. Piccart-Gebhart M, Holmes E, Baselga J, de Azambuja E, Dueck AC, Viale G, et al. Adjuvant lapatinib and trastuzumab for early human epidermal growth factor receptor 2-positive breast cancer: results from the randomized phase III adjuvant lapatinib and/or trastuzumab treatment optimization trial. J Clin Oncol. 2016;34:1034-42.

82. Vaz-Luis I, Ottesen RA, Hughes ME, Mamet R, Burstein HJ, Edge $\mathrm{SB}$, et al. Outcomes by tumor subtype and treatment pattern in women with small, node-negative breast cancer: a multi-institutional study. J Clin Oncol. 2014;32:2142-50.

83. Untch M, Jackisch C, Schneeweiss A, Conrad B, Aktas B, Denkert $\mathrm{C}$, et al. Nab-paclitaxel versus solvent-based paclitaxel in neoadjuvant chemotherapy for early breast cancer (GeparSepto-GBG 69): a randomised, phase 3 trial. Lancet Oncol. 2016;17:345-56.

84. Poggio F, Bruzzone M, Ceppi M, Pondé NF, La Valle G, Del Mastro L, et al. Platinum-based neoadjuvant chemotherapy in triplenegative breast cancer: a systematic review and meta-analysis. Ann Oncol. 2018;29:1497-508.

85. Masuda N, Lee S-J, Ohtani S, Im Y-H, Lee E-S, Yokota I, et al. Adjuvant capecitabine for breast cancer after preoperative chemotherapy. N Engl J Med. 2017;376:2147-59.

86. Natori A, Ethier J-L, Amir E, Cescon DW. Capecitabine in early breast cancer: a meta-analysis of randomised controlled trials. Eur J Cancer. 2017;77:40-7.

87. Runowicz CD, Leach CR, Henry NL, Henry KS, Mackey HT, Cowens-Alvarado RL, et al. American Cancer Society/American Society of Clinical Oncology Breast Cancer Survivorship Care Guideline. CA Cancer J Clin. 2016;66:43-73.

88. Sisler J, Chaput G, Sussman J, Ozokwelu E. Follow-up after treatment for breast cancer: practical guide to survivorship care for family physicians. Can Fam Physician. 2016;62:805-11.

89. Barnadas A, Algara M, Cordoba O, Casas A, Gonzalez M, Marzo $\mathrm{M}$, et al. Recommendations for the follow-up care of female breast cancer survivors: a guideline of the Spanish Society of Medical Oncology (SEOM), Spanish Society of General Medicine (SEMERGEN), Spanish Society for Family and Community Medicine (SEMFYC), Spanish Society for General and Family Physicians (SEMG), Spanish Society of Obstetrics and Gynecology (SEGO), Spanish Society of Radiation Oncology (SEOR), Spanish Society of Senology and Breast Pathology (SESPM), and Spanish Society of Cardiology (SEC). Clin Transl Oncol. 2018;20:687-94.

90. Curigliano G, Burstein HJ, Winer EP, Gnant M, Dubsky P, Loibl $\mathrm{S}$, et al. De-escalating and escalating treatments for early-stage breast cancer: the St. Gallen International Expert Consensus Conference on the Primary Therapy of Early Breast Cancer 2017. Ann Oncol. 2017;28:1700-12. 